\title{
La inserción laboral de los universitarios a través de las prácticas en empresas
}

Rafael Martínez Martín

Universidad de Granada

RESUMEN

Las prácticas en empresas para universitarios se regulan en el año 1981 (RD 1491/1981) con el objetivo de mejorar la formación práctica de los estudiantes de últimos cursos de carrera. Su implantación es una realidad en los años noventa con la implicación cada vez mayor de instituciones, empresarios y alumnos, que responde a la necesidad de acercar la universidad al mundo empresarial y de facilitar la inserción laboral de los universitarios. En el presente artículo se analizan la experiencia práctica en la empresa, opiniones y valoraciones de los universitarios sobre las prácticas en empresas en la Universidad de Granada y sus trayectorias laborales.

Palabras clave: Inserción profesional, Universitarios, Formación, Cualificaciones, Prácticas en empresas. 


\section{INTRODUCCIÓN}

El desempleo y la precariedad laboral, desde la década de 1980 en España, aparecen como problemas fundamentales que afectan a todos los grupos de población, aunque de forma notoria a los jóvenes menores de 25 años. La situación del mercado laboral ha mejorado de forma considerable desde el año 2000 , pero sigue constituyendo uno de los principales problemas de la sociedad.

En los últimos diecisiete años se han producido importantes transformaciones, como son: el desarrollo de procesos de mundialización y globalización económica, la modernización e introducción de nuevas tecnologías en el ámbito productivo, la creciente liberalización de los mercados y el crecimiento del sector servicios muy vinculado al turismo. Estos rasgos, característicos de la estructura productiva española, explican en cierta medida los elevados niveles de temporalidad en la contratación y las dificultades de acceder a un empleo de calidad.

El sector servicios crea el $90 \%$ de los nuevos puestos de trabajo; además, es un sector muy dependiente del turismo, con sus correspondientes ciclos de expansión y recesión a lo largo del año. La coyuntural demanda de empleo característica de este sector y la liberalización de las relaciones laborales constituyen factores estructurales determinantes de la actual realidad laboral española.

Son muchos los condicionantes que determinan la situación de la población activa en el mercado de trabajo, pero uno de los argumentos más evidentes y manejados es la necesidad económica y empresarial de incrementar los niveles de competitividad y productividad.

Los diferentes estudios sobre inserción laboral realizados en el contexto de la Unión Europea coinciden en señalar la importancia del nivel educativo en el desarrollo de los procesos de inserción profesional. Según muestra la Encuesta de Población Activa, a mayor nivel educativo, mejor es la situación laboral del sujeto. En general, están ocupados el $94 \%$ de los doctores y el $84 \%$ de los titulados universitarios, frente a los que poseen niveles de estudios inferiores, que soportan mayores tasas de desempleo y de precariedad laboral (EPA, 2.ำ trimestre 2002).

Como afirma Riviére (1997: 87), los titulados universitarios se incorporan más tarde al mercado laboral al dedicar más tiempo a la formación, pero cuando acceden lo hacen, en su mayoría, a puestos de trabajo de mayor categoría, estabilidad y adecuados a su formación.

Aunque ésta es la tendencia general que se aprecia en los titulados universitarios, se ha de tener presente que existen titulaciones con mayores posibilidades de acceso al mercado 
laboral que otras (García, 1998: 176-178; Masjuan y otros, 1996: 75-78; Fernández-Abascal, 1998: 181; Navarro, 1989: 138-139; SOPP, 1996: 74-86; Figuera, 1996: 148-150). En concreto, las áreas de Enseñanzas Técnicas y Ciencias Experimentales y de la Salud presentan mayores niveles de inserción profesional que Humanidades, Ciencias Jurídicas y Sociales y Enseñanzas Artísticas (MTAS, 1998: 45) ${ }^{1}$.

La recesión del empleo y de la calidad laboral, cuyo origen se sitúa en la crisis energética de 1973, han afectado a todos los grupos de población, incluidos a los de mayor nivel educativo. El título universitario ha dejado de garantizar por sí mismo el acceso al mercado laboral.

Los debates, críticas e investigaciones realizados al respecto, han intentado conocer las principales causas que explican el incremento del desempleo y de la precariedad laboral en los universitarios. En general, se destacan dos factores fundamentales:

El primero intenta justificar el desempleo de los universitarios aludiendo a su importante incremento en los últimos años, que ha hecho que el mercado laboral se sature y no pueda dar cabida a todos los titulados.

En base a esta hipótesis, la Teoría del Capital Humano, con plena vigencia en los años 1950-1960 y en un contexto caracterizado por la demanda creciente de mano de obra cualificada y el pleno empleo, pierde vigencia a la hora de explicar el notable incremento de universitarios. La inversión en educación deja de ser segura desde los años 1980, al contemplarse elevadas tasas de desempleo en los titulados universitarios. Sin embargo, su número ha seguido aumentando ante la necesidad de incrementar las credenciales (títulos universitarios, entre otros) para competir por los escasos puestos de trabajo, como afirman las Teorías Credencialistas.

El segundo de los debates se ha centrado en la calidad de la Universidad. La enseñanza universitaria ha sido calificada como anacrónica, poco práctica y alejada de las cualificaciones que demandan los empresarios. Este tipo de concepciones han creado estereotipos en los empleadores que les han llevado a priorizar la experiencia profesional a la titulación. Un

\footnotetext{
1 Según los datos del Ministerio de Trabajo y Asuntos Sociales (1998: 46), las ofertas de empleo dirigidas a los titulados provienen, en su mayoría, de las actividades económicas relacionadas con servicios a las empresas (informática, consultoría, asesoramiento y servicios técnicos de arquitectura e ingeniería), de otro tipo de servicios (inmobiliarias, intermediación financiera, comercio, hostelería y transporte), electricidad y electrónica, maquinaria, química, construcción y, en menor medida, de los sectores agroalimentario y de energía. Por áreas de enseñanza, seis de cada diez ofertas de trabajo corresponden al área de Ciencias Sociales y Jurídicas, seguida del área de Enseñanzas Técnicas (38\%). El resto de las áreas se reparten el escaso porcentaje que queda, Humanidades (1,7\%), Ciencias Experimentales y de la Salud $(0,45 \%)$ y Enseñanzas Artísticas $(0,28 \%)$.
} 
recién licenciado, sin experiencia laboral, conlleva costes adicionales en formación complementaria, restándole ocupabilidad².

Las medidas adoptadas para corregir estas deficiencias han sido diversas. Desde el replanteamiento de los planes de estudio hasta la creciente introducción de convenios entre las universidades y las empresas, para que los alumnos y titulados universitarios puedan realizar un periodo de prácticas en diferentes ámbitos laborales adecuados a su formación. Conjugar formación académica con experiencia profesional parece lo más adecuado.

Las prácticas en empresas tienen como principal objetivo mejorar la formación práctica de los universitarios y adecuar, en mayor medida, su formación a las demandas del mercado laboral. La mejora de las cualificaciones ${ }^{3}$ es su cometido principal, pero los universitarios y la propia dinámica de los programas de prácticas van más allá al perseguir el inicio de la transición a la vida activa ${ }^{4}$ y su posterior inserción laboral plena ${ }^{5}$.

La inserción laboral, por tanto, se ha convertido en uno de los principales objetivos a conseguir con la realización de prácticas en empresas. La inmensa mayoría de los universitarios de la Universidad de Granada lo constatan a la hora de solicitarlas y realizarlas. De hecho, constituyen una buena oportunidad, al mejorar las credenciales, las redes sociales e incorporarse a las ventajas que proporcionan los mercados internos de trabajo ${ }^{6}$.

2 La ocupabilidad hace referencia a la probabilidad que tiene un individuo de encontrar un trabajo en un momento dado (Blanch, 1990: 183).

3 Por cualificación profesional se entiende la conjugación de títulos, certificados y acreditaciones de la experiencia laboral, para referirla a la competencia personal necesaria al buen desempeño de un trabajo (Retuerto, 1997: 103).

${ }^{4}$ El concepto de transición hace alusión a un proceso de cambio que tiene lugar a lo largo de la vida del individuo, que requiere una reflexión personal (historia personal y profesional) y contextual (contexto socioprofesional) y que se sustenta en una información suficiente, en una actitud positiva y en la adquisición de unas destrezas adecuadas (Auberni, 1995: 396). Este concepto incluye dos procesos interrelacionados entre sí: la transición a la vida activa y la transición a la vida adulta. Mientras que la transición a la vida activa hace alusión al paso de la escuela al trabajo, que culmina con la inserción laboral y el consiguiente desempeño de una profesión, la transición a la vida adulta es un proceso complejo que tiene lugar con la incorporación del joven a la vida adulta y donde confluyen múltiples factores de carácter evolutivo, madurativo, social y económico (ibídem: 397). Cuando ambos procesos culminan se ha conseguido la inserción socioprofesional. En este sentido, Casal (1997) considera que las dificultades en el proceso de transición es el hecho social más significante de los años setenta en relación a los jóvenes, al constatarse elevadas tasas de paro juvenil y el aumento del tiempo de espera entre el abandono o finalización de la escolarización y la inserción profesional plena.

5 La inserción laboral supone el acceso al empleo y el desarrollo de trayectorias laborales que han de desembocar, en teoría, en una inserción laboral plena. Por inserción laboral plena se entiende la situación en la cual se adquiere un trabajo a tiempo total, estable y acorde con la formación adquirida (Figuera, 1996: 129).

6 Doeringer y Piore (1983b: 324) definen los mercados internos de trabajo como aquellos donde el precio y la asignación del trabajo son regidos por un conjunto de normas y procedimientos administrativos, frente al mercado externo de trabajo, donde los precios y la asignación de formación son controlados de forma directa por variables económicas. Los trabajadores internos tienen determinados derechos y privilegios como son: continuidad en el empleo, competencia protegida del mercado externo 
Entre los factores más determinantes para conseguir un empleo se encuentra la experiencia profesional. La falta de experiencia profesional es considerada como la segunda dificultad más importante para el acceso al trabajo por parte de los licenciados en Ciencias Políticas y Sociología (Latiesa y otros, 1996: 58). Además, es el requisito de acceso al empleo nombrado con más frecuencia (74\%) por los estudiantes universitarios (González, 1998: 131).

Los universitarios consideran al respecto (COIE, 1995: 8):

No me extraña que cuando vas a una empresa a pedir trabajo te digan: tiene usted experiencia, porque si es que no tiene, tendrá el título, pero el título no vale para nada. ... normalmente te exigen conocimientos de algo muy específico que no se aprende en la carrera.

Estas manifestaciones, extraídas de un grupo de discusión, plasman las dificultades de acceso al mercado laboral de los titulados universitarios que no tienen experiencia laboral. La credencial universitaria ha perdido valor por sí sola al disminuir buena parte de su poder de ocupabilidad.

Tener experiencia es clave, no sólo por el nivel de especialización que conlleva, sino también por la importancia del capital relacional en el mundo del trabajo ${ }^{7}$.

Los universitarios opinan sobre el tema (ibídem):

A la empresa privada si no vas con padrino... yo la gente que conozco que ha entrado, ha entrado a base de conocer a alguien. Está claro que necesitas un padrino para entrar en la empresa privada, si tu padre no tiene medios o no tiene contactos...

y derechos exclusivos sobre los puestos cubiertos internamente. Las vacantes se suelen cubrir con la promoción interna o transferencia de los trabajadores que hayan conseguido entrar. Estos mercados están presentes en todas las economías y su origen y desarrollo se debe a tres factores: la costumbre, la cualificación específica en el trabajo y la formación en el trabajo (ibídem: 346).

7 La Teoría del Capital Relacional establece que los canales de acceso al empleo (amigos, parientes o conocidos) son muy eficaces a la hora de encontrar un empleo, aunque su efectividad depende de distintas circunstancias; entre ellas, el tipo de trabajo y de empresa a donde se pretende acceder. Mientras en las pequeñas y medianas empresas se accede principalmente a través de contactos personales, en las empresas públicas el mecanismo de acceso suele ser a través de canales oficiales formalizados (Requena, 1991: 76).

Los costes de búsqueda y acceso al empleo (movilidad, información, etc.) se ven ampliamente reducidos con las redes sociales (amigos, familia, vecinos, etc.). En esta línea, Doeringer y Piore (1983: 312) consideran que la aceptación del nuevo empleado por parte de sus compañeros en la empresa es fundamental para poder adquirir determinadas cualificaciones necesarias para desarrollar el trabajo. Por tanto, la aceptabilidad social es clave en el proceso de obtención de cualificaciones, y esta aceptación es sensible a factores como la raza, sexo y creencias sociales compartidas (ibídem). 
Estas relaciones pueden provenir de la experiencia laboral previa, de la posición de clase familiar o del medio biográfico en la estructura social. El status adscrito tiene su reflejo en la posesión de mayor capital relacional y mejor posición económica, que les permiten acceder a una formación de elite. La realización de másters, de cursos específicos, la formación en el extranjero, la movilidad geográfica y la posibilidad de poder dedicar más tiempo a la búsqueda de un trabajo, determinan una inserción laboral de mayor calidad. Esta visión estructuralista, otorga especial importancia al origen de clase social como factor determinante de los procesos de inserción profesional.

\section{INSERCIÓN LABORAL Y PRÁCTICAS EN EMPRESAS PARA UNIVERSITARIOS}

La formación está jugando un papel importante en la lucha contra el paro y en el desarrollo de las economías. Cada vez son más numerosas las fórmulas que procuran imbricar la formación en el aula con la formación en la empresa ${ }^{8}$. Entre ellas, la concesión de ayudas, subvenciones o deducciones fiscales a los empresarios para formar a jóvenes y la creación de nuevas formas de contratación. Estas medidas se han utilizado en distintos países, como Bélgica, Francia, Grecia, Italia, Portugal y España, como medios de lucha contra el paro. La oportunidad de comenzar a trabajar y de adquirir diferentes cualificaciones en el lugar de trabajo, resulta una fórmula eficaz de inserción laboral. De hecho, el $12 \%$ de los contratos para la formación y el $28 \%$ de los contratos en prácticas, se convierten en contratos fijos (OCDE, 1998: 224).

Los sistemas de formación a nivel internacional destacan la importancia de la formación adquirida en la empresa. Las universidades, concienciadas de la necesidad de adecuar la formación a las nuevas demandas empresariales, han desarrollado programas de prácticas encaminados a favorecer la interacción de la universidad con la empresa.

Las prácticas en empresas son demandadas de forma creciente por jóvenes que ven terminados sus estudios sin haber llevado a cabo los conocimientos adquiridos. El contacto con el mundo del trabajo establece una fractura con la formación de laboratorio para llevarla a la realidad. En muchas ocasiones se rompe con mitos asentados durante la carrera sobre la utilidad, las salidas profesionales y el papel a desempeñar en el trabajo.

\footnotetext{
8 Los países de la OCDE impulsan, de forma creciente, medidas de creación directa de empleo. Entre ellas los contratos de empleo solidario en Francia, dirigidos a sectores de población desfavorecidos que facilitan su inserción laboral a través de contratos subvencionados por el Estado. También el programa sueco de iniciación práctica al trabajo, dirigido a jóvenes con edades comprendidas entre los 18 y 25 años.
}

Desde el año 1991, los Países Bajos tienen un programa de creación directa de empleo llamado garantía de empleo para los jóvenes, que ofrece empleos en el sector público, privado y en el tercer sector o de voluntarios (OIT, 1998: 224-225). 
Los universitarios que las han realizado están en una situación de ventaja frente a otros candidatos a un primer empleo. La labor de orientación profesional que aportan sobre los sectores más dinámicos, las demandas de los empresarios y los mecanismos de búsqueda, junto con las oportunidades de establecer contactos personales, son factores muy relevantes para la consecución de la inserción profesional.

Los diferentes estudios señalan que se han convertido en un referente importante en el currículum de los recién titulados a la hora de conseguir trabajo. Además, para muchos han supuesto el primer contacto con el mundo del trabajo, el inicio de la carrera profesional y un puente directo a la inserción profesional.

La investigación llevada a cabo por Latiesa y otros (1996: 62-66) muestra que el 61\% de los sociólogos y politólogos las solicitaron como medio de encontrar un trabajo. Las prácticas son consideradas como una de las vías principales de acceso al empleo; en el primer trabajo relevante en su vida que describen los licenciados, la situación más frecuente es que sean trabajadores en prácticas o colaboradores. Además, una proporción mayoritaria las considera fundamentales como medio de mejorar su formación. De forma textual argumentan (ibídem: 82):

Incluiría un amplio programa de prácticas que permitieran, sobre la marcha, ver la aplicabilidad de los estudios teóricos al mundo real laboral.

En otros estudios promovidos por el COIE (1997: 67-68) se constata la necesidad de adecuar la formación a las nuevas demandas empresariales. Los resultados señalan que la mayoría del profesorado (65\%) considera que los titulados no están lo suficientemente preparados para satisfacer las demandas del mundo empresarial, y que el principal motivo obedece a la escasa proyección práctica de la formación.

La distancia entre la universidad y la empresa puede ser reducida con las prácticas en empresas. Dotar a la formación académica de experiencia laboral es una forma de adaptar la formación a las demandas empresariales y de incrementar el grado de ocupabilidad de los titulados. Los empresarios han manifestado la importancia de la experiencia profesional frente a la titulación universitaria. La concepción generalizada de que el recién licenciado no posee las destrezas y conocimientos adecuados al mundo de la empresa ha hecho que la experiencia laboral adquiera mayor peso que la titulación a la hora de contratar a alguien.

Desde que se iniciaran las prácticas en empresas en las Escuelas Politécnicas, después en los estudios empresariales y en la actualidad en todas las ramas laborales, su demanda 
e implantación ha ido en aumento. En España, las prácticas en empresas para estudiantes universitarios están reguladas por el Real Decreto 1497/1981, de 19 de junio, sobre Programas de Cooperación Educativa, y por el Real Decreto 1845/1994, de 9 de septiembre, que adapta el anterior a los planteamientos de la reforma de las titulaciones universitarias. Según esta legislación, las universidades pueden establecer Programas de Cooperación Educativa mediante convenios con empresas para la formación especializada y práctica de los alumnos de los dos últimos cursos de carrera o aquellos que hayan completado el $50 \%$ de los créditos de la titulación. La duración no puede exceder del 50\% del tiempo íntegro del curso académico. En lo que respecta a la remuneración, no existe como tal y sí como ayuda económica, cuyo objetivo es sufragar los gastos que la realización de las mismas puedan ocasionar al alumno. Tampoco han de suponer una vinculación laboral, ya que los únicos compromisos que han de asumir empresas y alumnos son los estrictamente estipulados en el convenio establecido entre la universidad y la empresa.

Entre las instituciones que se preocupan de fomentarlas se encuentran: el Instituto $\mathrm{Na}$ cional de Empleo, financiando programas de formación en alternancia destinados a proporcionar cualificación práctica a estudiantes universitarios de últimos cursos de carrera y a insertar a los estudiantes en el mercado laboral; las universidades, a través de convenios con los empresarios y con su inclusión en los planes de estudios; la Fundación Universidad-Empresa, convocando prácticas dirigidas específicamente al área de Humanidades para dar la oportunidad de encontrar el primer empleo a estudiantes de titulaciones en menor medida demandadas por el mercado; el Círculo de Progreso, con un servicio personalizado de ofertas; e Infoempleo, orientando a los universitarios para poder realizarlas en el extranjero.

Dentro de la Formación Profesional tienen especial relevancia, ya que se encuentran en todos los ciclos formativos. El Programa de Formación Profesional en España (1998-2002) pretende estrechar los lazos entre la formación y la empresa. Las prácticas surgen como un medio de conseguirlo, bajo la idea de aprovechar el papel de la empresa como organismo capaz de facilitar el aprendizaje, la profesionalización y la inserción laboral, a través de la formación en centros de trabajo. Entendido así, vienen a sustituir a la figura del aprendiz, al constituir un ejemplo válido de lo que se ha denominado formación en alternancia.

El modelo de política formativa hacia el que se dirige la Unión Europea no olvida la importancia de la formación práctica en las empresas. Muestra una síntesis de los tres modelos europeos representativos, como son el germánico (donde la empresa ha asumido el papel formativo de los gremios), el británico (basado en la competencia profesional) y el francés (fundamentalmente académico). Pretende conseguir un sistema de formación general aplicable a distintas profesiones o puestos, que pone el acento en competencias de aplicación 
en distintas situaciones de trabajo, además de contar con una unidad orientadora para la construcción de itinerarios formativos adecuados a los requerimientos del mercado laboral (García, 2000: 44).

A continuación se exponen los principales resultados empíricos de la investigación llevada a cabo en la Universidad de Granada sobre la incidencia de las prácticas en empresas en los procesos de inserción laboral de los universitarios.

Para la evaluación de las prácticas en empresas se han utilizado técnicas de recogida de información cualitativas, como el grupo de discusión y el análisis documental, y cuantitativas, como ha sido la encuesta y el análisis de datos primarios. Las técnicas cuantitativas han tenido un papel preponderante, ya que la encuesta ha sido la principal técnica utilizada para evaluarlas.

La población objeto de estudio la compone un total de 2.937 individuos, que constituyen la totalidad de los universitarios que han realizado prácticas en empresas, desde el año 1991 hasta el año 2000, a través de la Oficina de Prácticas de la Universidad de Granada.

El cuestionario ha sido suministrado por correo en los domicilios de los universitarios previa llamada telefónica, durante los meses de mayo, junio y julio de 1999 y septiembre y octubre de 2000. El nivel de respuesta ha sido del 53\%, que le corresponde una muestra total de 414 encuestas. En el supuesto de que el muestreo hubiera sido aleatorio, al tamaño de la muestra global obtenida asume un nivel de error de $\pm 3 \%$, para un nivel de confianza del $95,5 \%$ (2 sigmas) y siendo $P=Q$.

Entre los principales objetivos de la investigación se encuentra el análisis de las trayectorias laborales ${ }^{9}$ que han descrito los universitarios antes y después de realizar las prácticas

9 Spilerman (1977; cit. García, 1998: 49) establece el concepto de línea de carrera para referirse a las estructuras relativamente estables del mercado de trabajo a través de las cuales se mueven los trabajadores. Las trayectorias laborales constituyen las líneas de carrera de los individuos, y el conjunto de trayectorias laborales por las que transcurre su vida forman su carrera laboral.

Las carreras son vistas como una secuencia de posiciones ocupadas por una persona durante el transcurso de su vida (Poole et al., 1993: 40).

La teoría de las transiciones del rol laboral mantiene que la entrada a un nuevo rol produce un desarrollo personal y/o un desarrollo de rol. El desarrollo personal es una función de la novedad de un rol y el deseo de esa novedad de retroalimentarse, mientras que el desarrollo del rol es una función discrecional (la discrecionalidad se refiere a la libertad de cada uno para variar las características de la tarea; una baja discrecionalidad conlleva pocas oportunidades para modificar el propio rol, y a la inversa). En este sentido, las carreras son definidas como

... una secuencia de roles laborales o como una secuencia de experiencias de trabajo de una persona a lo largo del tiempo. Lo que cada secuencia de actividades tiene en común es la experiencia de la transición: el proceso de sepa- 
en empresas, con la pretensión de conocer en qué medida las prácticas han sido determinantes en la configuración de sus carreras laborales.

En primer lugar se describen los trabajos remunerados que han desempeñado previos a las prácticas en empresas, para pasar a estudiar los itinerarios laborales posteriores a las prácticas, ya sean en la misma empresa donde las realizaron, que supone la continuidad de la relación laboral, o en otros ámbitos laborales, al romper los vínculos con la empresa donde realizaron sus prácticas.

\section{EL INICIO DEL PROCESO DE TRANSICIÓN A LA VIDA ACTIVA EN LOS UNIVERSITARIOS}

En este apartado se estudian las situaciones laborales antes de realizar las prácticas en empresas. Se pretende conocer en qué medida las prácticas constituyen la primera experiencia laboral de los universitarios y analizar sus situaciones laborales, con el propósito de describir sus carreras laborales (figura 1).

La mayoría $(63,6 \%)$ de los universitarios no han realizado trabajos remunerados antes de las prácticas. No obstante, una proporción considerable afirma haberlos realizado $(36,4 \%)^{10}$, sin que existan destacadas diferencias estadísticas por sexo o nota media en la carrera, aunque sí las hay por áreas de estudio. Las áreas de Humanidades y Ciencias Jurídicas y Sociales presentan las mayores proporciones de universitarios que trabajan antes de realizar las prácticas, frente a Ciencias de la Salud, Experimentales y Enseñanzas Técnicas, con menor representatividad. La menor relevancia de estas áreas se explica por las mejores expectativas laborales que presentan las titulaciones que comprenden. El alumnado suele dedicar todo su tiempo a los estudios, para alcanzar mayores niveles de competitividad ante las posibilidades profesionales que se le brindan.

La inmensa mayoría de los que han trabajado $(84,1 \%)$ han tenido entre uno y tres empleos, y el 5,9\% ha desempeñado más de tres, siendo la media de empleos desempeñados de 2,3 .

La calidad de los empleos es en general baja, ya que una importante proporción $(33,8 \%)$ ha trabajado sin contrato, el $43,6 \%$ con contratos temporales y el $12,6 \%$ con contratos de

rarse de los roles anteriores y de unirse a nuevos roles. Las transiciones de rol laboral a menudo suponen una reorientación de metas, actitudes, identidad, rutinas de comportamiento, redes informales y muchos otros pequeños cambios. Así, las transiciones de rol laboral constituyen un periodo de discontinuidad y flujo donde los individuos y sus roles deben discurrir hacia una nueva sincronización (traducción mía) (Ashforth y Saks, 1995: 15).

10 Por trabajo se entiende cualquier actividad remunerada, aunque sea esporádica. 
FIGURA 1

Situación laboral de los universitarios antes de realizar las prácticas

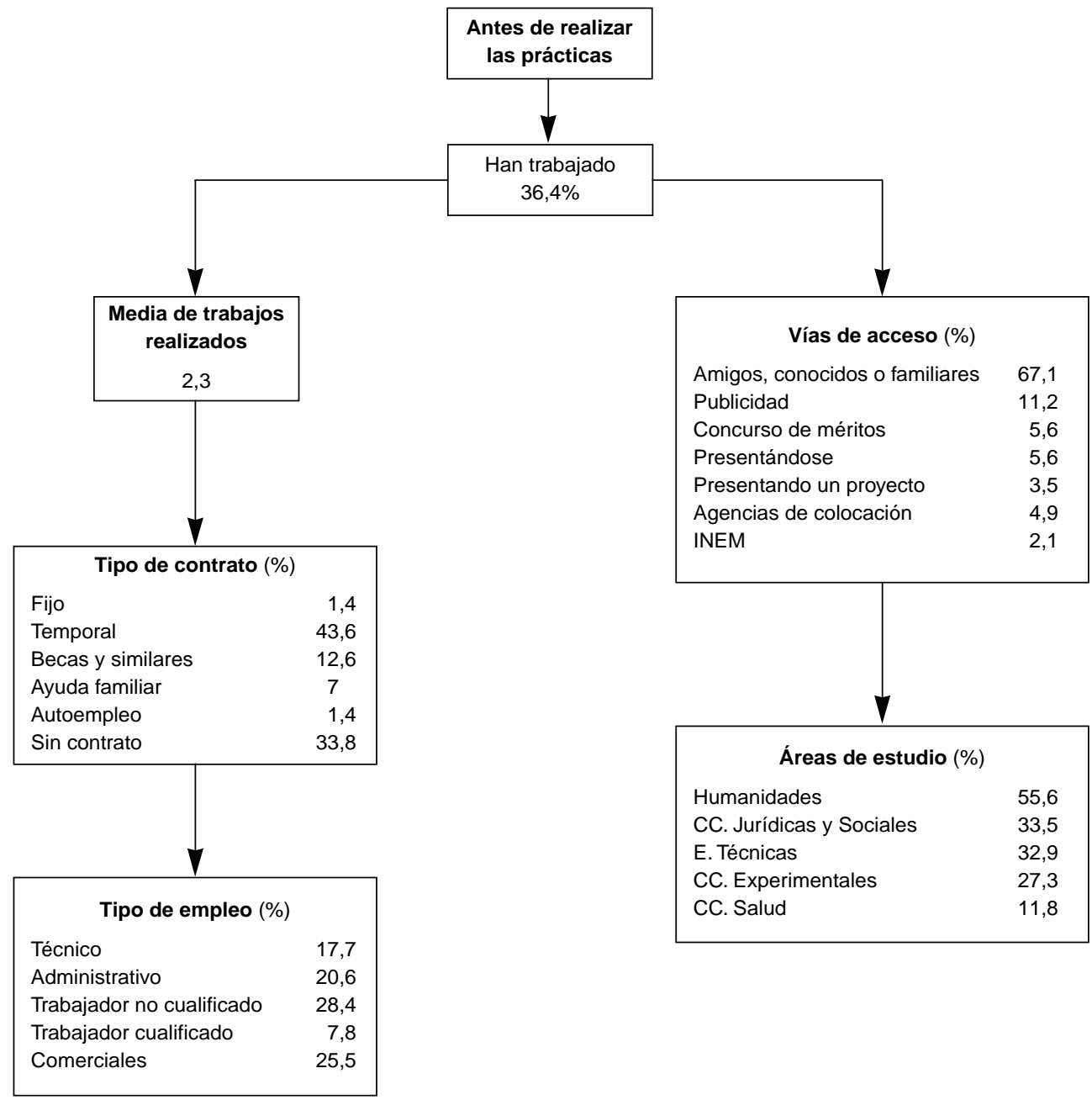

FUENTE:

Elaboración propia.

formación, prácticas, becas o similares. Una minoría $(1,4 \%)$ ha tenido un trabajo fijo a tiempo parcial, sin que existan contratos indefinidos a tiempo total; el $7 \%$ ha desarrollado labores de ayuda familiar, y el 1,4\% ha recurrido al autoempleo. 
Lo predominante es la temporalidad en la contratación, al ser uno de los principales rasgos que caracterizan al mercado laboral español desde mediados de los años ochenta. A esta circunstancia habría que añadir la situación de los universitarios que compaginan estudios con trabajo, con la consiguiente predilección por un empleo a tiempo parcial.

En lo que respecta a la categoría profesional, el 17,7\% ha trabajado como técnico superior o medio, siendo una proporción relativamente elevada al ser universitarios que no han terminado sus estudios. El resto de los empleos que han ocupado son de trabajador no cualificado en el sector industrial o de servicios $(28,4 \%)$, de comerciales o vendedores $(25,5 \%)$, de administrativos $(20,6)$ y de trabajadores cualificados en el sector industrial o de servicios $(7,8 \%)$.

La vía de acceso al trabajo ha sido para la mayoría a través de amigos, conocidos o familiares $(67,1 \%)$; a una distancia considerable, le siguen la información adquirida por medio de anuncios publicitarios $(11,2 \%)$, los concursos de méritos $(5,6 \%)$ y presentándose de forma espontánea (5,6\%). En proporciones inferiores, lo consiguieron presentando un proyecto $(3,5 \%)$ o a través de las Agencias de Colocación o de las ETT (4,9\%). Cabe destacar el escaso protagonismo del INEM, ya que sólo un 2,1\% lo ha conseguido por medio de esta institución.

Las redes sociales desempeñan un papel primordial a la hora de acceder al mercado de trabajo, como se puede apreciar a la luz de estos resultados. Amigos, familiares y conocidos aportan la información necesaria a los universitarios y empresarios, convirtiéndose éstos en la principal vía de acceso al empleo.

En otros estudios realizados se han encontrado niveles similares de actividad laboral antes de terminar los estudios. En concreto, el $37 \%$ de los universitarios de últimos cursos de carrera de la Universidad de Huelva han desarrollado actividades laborales durante sus estudios, por lo general ocupaciones de carácter esporádico (Cruz y otros, 1999: 161); en la Universidad Carlos III de Madrid, la proporción de estudiantes que trabajan durante la carrera disminuye de forma considerable, ya que el 14\% tenía algún trabajo remunerado antes de terminar la carrera (SOPP, 1996: 63); y en Palencia, el 29,8\% de los jóvenes que cursan estudios universitarios realizan actividades remuneradas (Fernández-Abascal, 1998: 123).

\section{LAS TRAYECTORIAS LABORALES EN LAS EMPRESAS DONDE REALIZARON LAS PRÁCTICAS}

Una vez estudiada la situación profesional antes de realizar las prácticas, interesa conocer qué ha ocurrido con los universitarios que al terminar las prácticas siguen trabajando en la empresa donde las realizaron (figura 2). 
FIGURA 2

Situación laboral al terminar las prácticas en las empresas donde las realizaron

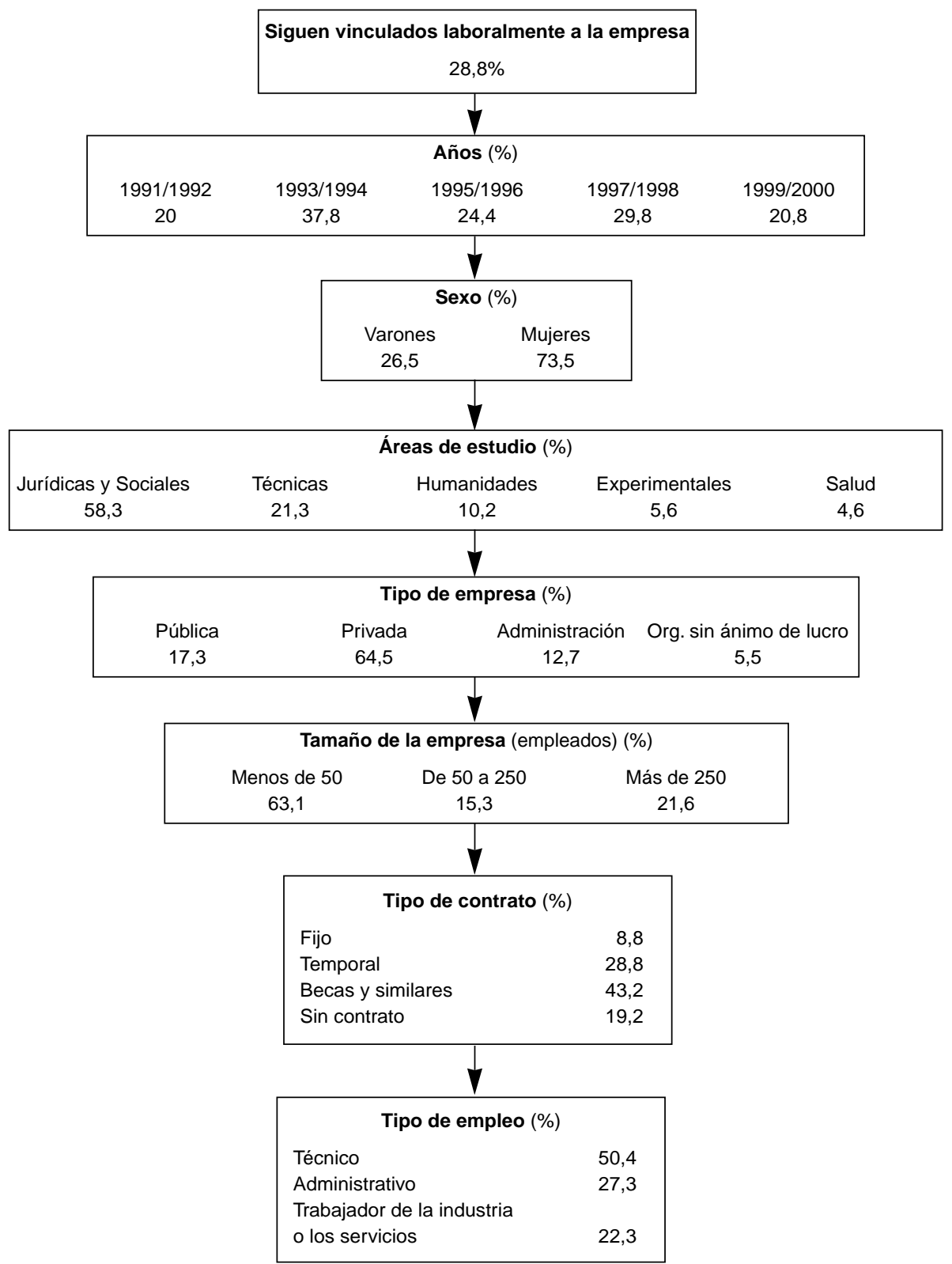

FUENTE:

Elaboración propia. 
Al terminar las prácticas, la mayoría de los universitarios mantuvieron algún tipo de vínculo con la empresa donde las realizaron, ya sea de amistad $(43,4 \%)$ o laboral $(28,8 \%)$. No obstante, tres de cada diez universitarios $(28,2 \%)$ perdieron el contacto total con la empresa al terminarlas.

El aspecto más relevante, a la hora de analizar sus trayectorias profesionales, son los vínculos laborales. Los niveles de vinculación, al terminar las prácticas, se sitúan en el $28,8 \%$ para la totalidad de universitarios. Esta proporción ha mostrado una tendencia creciente desde sus inicios. En concreto, de los que las realizaron en el curso 1991/92, el 20\% mantuvo los vínculos laborales al terminarlas; en el curso 1993/94, la proporción se eleva de forma considerable para situarse en el 37,8\%; en el curso 1995/96 desciende la proporción hasta el $24,4 \%$; en el curso $1997 / 98$ vuelve a elevarse hasta el $29,8 \%$; y en el 1999/2000 se sitúa en el $20,8 \%$.

Las proporciones de universitarios que han mantenido los vínculos laborales al terminar las prácticas varían en función del sexo, área de estudio y del tamaño y tipo de empresa, sin que existan diferencias significativas según la nota media en la carrera. La mayoría son mujeres, con titulaciones pertenecientes a las áreas de Ciencias Jurídicas y Sociales y Enseñanzas Técnicas, que han realizado sus prácticas en empresas privadas y con menos de 50 trabajadores. El papel destacado de la empresa privada se puede explicar en función de los procedimientos de contratación, mucho más espontáneos en la empresa privada, frente a la pública y la Administración, donde la dotación de nuevos puestos de trabajo suele acarrear dilatados trámites administrativos.

En otros estudios, los niveles de contratación posterior en las empresas donde realizaron las prácticas se sitúan en el 32,7\% (Martínez y Valero, 1999: 39); la investigación llevada a cabo por la Fundación Carlos III de Madrid señala que el $50 \%$ de los licenciados o diplomados logran un empleo estable en la empresa donde las realizaron (SOPP, 1996: 30); el estudio del profesor Delgado muestra que el $72 \%$ de los licenciados consiguieron su primer empleo gracias a las prácticas (García y Pastor, 1999: 81-92).

Las transformaciones experimentadas por el mercado laboral en las dos últimas décadas constatan que el hecho de encontrar un trabajo no significa haber conseguido una inserción laboral de calidad. Por ello es fundamental conocer su situación laboral, al objeto de valorar en qué medida han conseguido consolidar el proceso de inserción profesional.

La mayoría de los universitarios que siguieron vinculados a la empresa donde realizaron sus prácticas describen una situación precaria. En concreto, el 43,2\% mantiene la vinculación a través de becas y similares, el $28,8 \%$ con un contrato temporal, el 19,2\% trabaja sin 
contrato y el $8,8 \%$ tiene un contrato fijo. En lo referente al tipo de empleo, la mayoría ocupa puestos de técnicos medios o superiores $(50,4 \%)$, le siguen de administrativo $(27,3 \%)$ y, en menor medida, de comerciales o vendedores y de trabajadores de la industria o los servi$\operatorname{cios}(22,3 \%)$.

Aunque la situación es claramente precaria, la mayoría de los empleos que ocupan exigen formación universitaria, al desarrollar principalmente tareas de técnico medio o superior. Las posibilidades de consolidar su situación laboral a corto y medio plazo se han de tener presentes. Las trayectorias que suelen describir los universitarios son las de aproximación sucesiva; una vez que han conseguido iniciar su transición a la vida activa, van ganando posiciones en el mercado de trabajo hasta conseguir la inserción laboral plena $^{11}$.

En la actualidad (julio de 2000), transcurrido más o menos tiempo (entre uno y nueve años) en función del año en que realizaron las prácticas, casi la mitad de los que al terminar las prácticas quedaron vinculados a la empresa han perdido esta vinculación (figura 3).

Conforme se incrementa el tiempo transcurrido, las proporciones de los que siguen vinculados descienden de forma significativa. Los que realizaron las prácticas en los años 1991/92 y 1993/94 son los que menor proporción de vinculación laboral presentan en la actualidad, frente a los universitarios que las realizaron en los años 1995/96, 1997/98 y 1999/2000, que muestran proporciones muy superiores. Para la totalidad de los universitarios, en el momento de terminar las prácticas la continuidad en la empresa fue del $28,8 \%$; esta proporción disminuye hasta el $16 \%$ en la actualidad.

La significativa dinámica laboral de rotación externa que caracteriza al mercado laboral en España explica los diferentes niveles de permanencia en la empresa.

Un aspecto a destacar es la considerable mejora global de las condiciones laborales. La situación actual muestra un aumento de la estabilidad laboral, al incrementarse al 20,9\% los contratos indefinidos, y una mejora considerable del tipo de empleo, al disminuir la representatividad de las categorías laborales más bajas y elevarse hasta el 62,1\% la proporción de universitarios que trabajan como técnicos.

\footnotetext{
11 Las investigaciones llevadas a cabo por el GRET (Grup de Recerca Educació i Treball) de la Universidad Autónoma de Barcelona ponen de manifiesto la existencia de seis grandes modalidades de trayectorias laborales, entre ellas las trayectorias de aproximación sucesiva, caracterizadas por ser una modalidad de transición basada en el ensayo-error, donde el individuo transcurre por multitud de situaciones (empleo, paro, formación, subocupación, etc.), donde el ajuste de expectativas laborales también es continuo sobre la base de los resultados alcanzados y otros factores psicosociales. Supone el retraso de la emancipación familiar y es el modo dominante de transición desde la década de los ochenta (Casal, 1997). Estas trayectorias las suelen describir los universitarios.
} 


\section{FIGURA 3}

Situación laboral actual (julio 2000) en las empresas donde realizaron las prácticas

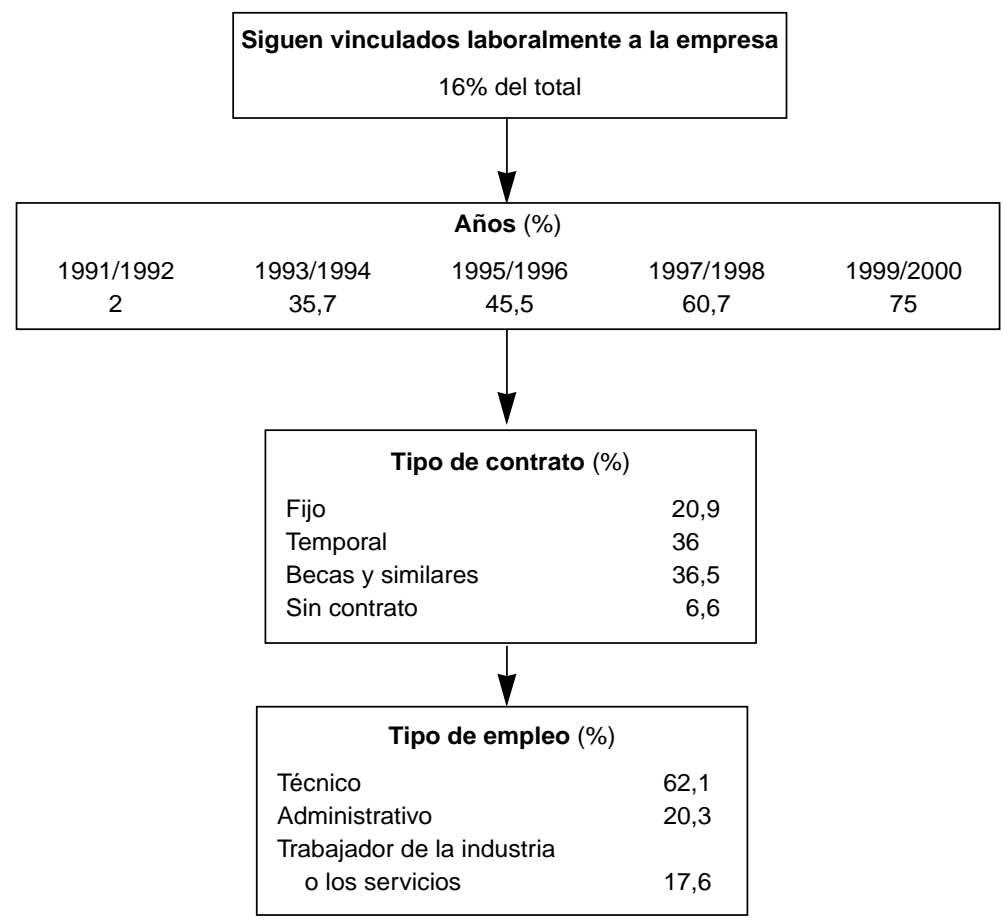

FUENTE:

Elaboración propia.

Entre las variables que se han contemplado en el análisis de las trayectorias laborales de los universitarios están la situación laboral y el nivel educativo de los padres. En general, existe una correlación positiva entre la categoría profesional actual de los universitarios y el nivel educativo y tipo de empleo de los padres. Las teorías de base marxista, como las de la correspondencia, se ven reflejadas en este estudio, al igual que en otros muchos. El estudio de Masjuan y otros (1996: 79), sobre los titulados universitarios en Barcelona, constata que las mujeres y los hijos de clases trabajadoras tienen más posibilidades de sufrir precariedad laboral que los de otras clases más altas; y la investigación sobre el mercado laboral de los jóvenes en Palencia (Fernández-Abascal, 1998: 145) concluye que los jóvenes de clases bajas tienen periodos de paro más prolongados y cuando acceden a un empleo lo hacen en peores condiciones, al sufrir en mayor medida la precariedad laboral. 


\section{LOS ITINERARIOS LABORALES DE LOS UNIVERSITARIOS QUE PERDIERON LOS VÍNCULOS LABORALES CON LA EMPRESA DONDE REALIZARON LAS PRÁCTICAS}

En el epígrafe anterior se han analizado las trayectorias de los universitarios que quedaron vinculados laboralmente a la empresa donde realizaron las prácticas. Sus líneas de carrera muestran, en general, una continuidad y una mejora de las condiciones laborales conforme pasan los años. Pero éstos representan una minoría (28,8\%), ya que la mayoría $(71,2 \%)$ perdió los vínculos laborales con la empresa al terminar las prácticas. A continuación se analizan las trayectorias laborales de los universitarios que, tras terminar las prácticas se ven obligados a emprender una nueva línea de carrera (figura 4).

La mayoría de los universitarios (63,3\%) al terminar las prácticas quedaron desempleados. Frente a éstos, una proporción considerable $(36,7 \%)$ encontró otro trabajo de forma inmediata. No existen diferencias estadísticas significativas por sexo, año en que realizaron las prácticas, nota media en la carrera y profesión o nivel educativo de los padres; sin embargo, en función del área de estudio existen diferencias considerables.

En concreto, la mayoría de los universitarios que han quedado desempleados al terminar las prácticas pertenecen al área de Ciencias Jurídicas y Sociales $(50 \%)$, les siguen los de Humanidades (20,4\%), Enseñanzas Técnicas (15,7\%), Ciencias Experimentales (10,9\%) y, por último, Ciencias de la Salud (3\%). Las menores probabilidades de empleo que presentan las áreas de Humanidades y Ciencias Jurídicas y Sociales quedan reflejadas en la situación de desempleo que, en mayor medida, experimentan los que han cursado carreras pertenecientes a estas áreas.

Una vez transcurridos una media de 5,2 meses en situación de desempleo, casi la mitad $(49,4 \%)$ encontró un trabajo. Las diferentes variables utilizadas muestran: que las mujeres encontraron trabajo en mayor proporción $(58,8 \%)$ que los varones $(41,2 \%)$; que conforme se incrementa el tiempo transcurrido desde que terminaron las prácticas, la proporción de universitarios que afirman haber realizado otros trabajos se incrementa, hasta llegar al $100 \%$ de los que las hicieron en el curso 1991/1992 ${ }^{12}$; y que existe una correlación positiva

\footnotetext{
12 La Teoría de la Búsqueda de Trabajo, desarrollada por Charles Holt y sus colegas del Urban Institute de Washington, considera que el paro es el resultado del proceso de búsqueda de trabajo en el que los trabajadores tienen una información limita$\mathrm{da}$, incertidumbre o falsas expectativas sobre el mercado de trabajo. El desajuste que pudiera producirse entre sus expectativas laborales y el trabajo que logran encontrar puede provocar el rechazo de determinados trabajos, para continuar la búsqueda de otro empleo más adecuado a sus aspiraciones y expectativas. La experiencia de la búsqueda traerá consigo un ajuste mayor entre sus expectativas y la realidad laboral, para terminar aceptando un trabajo o abandonando el mercado laboral. Para este enfoque, el paro es temporal (Doeringer y Piore, 1983: 310). Desde esta Teoría, la falta de información es el principal obstáculo de acceso al mercado de trabajo. La Teoría no se preocupa, al igual que la Teoría del Capital Humano, por los factores sociales que afectan a la elección del trabajador.
} 


\section{FIGURA 4}

Situación laboral de los universitarios que al terminar las prácticas perdieron los vínculos profesionales con la empresa donde las realizaron

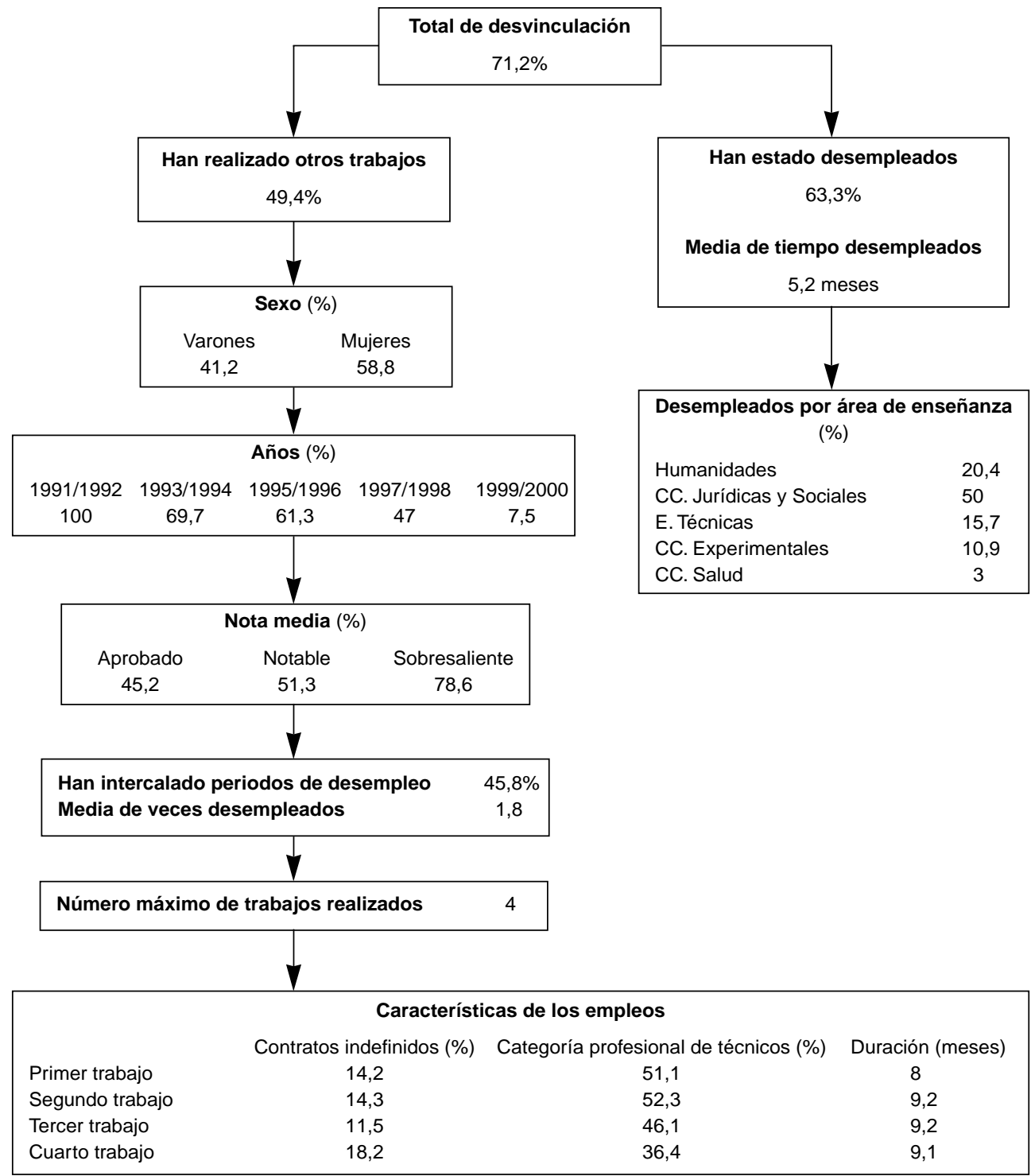

FUENTE:

Elaboración propia. 
entre la nota media y la proporción de universitarios que han trabajado después de las prácticas.

El número máximo de trabajos que llegaron a realizar es de cuatro y una considerable proporción $(45,8 \%)$ ha intercalado periodos de desempleo, siendo la duración media de los empleos de nueve meses. En concreto, la mayoría (80,5\%) ha estado desempleado una o dos veces y el $19,5 \%$ tres o más veces. La rotación empleo-paro-formación-empleo se pone de manifiesto en las trayectorias laborales que describen.

En cuanto a la estabilidad laboral a lo largo de las trayectorias laborales descritas, conforme pasan los años se aprecia un incremento de la estabilidad, que se traduce en un aumento de la contratación indefinida y un incremento también de la contratación temporal, que responde al notable descenso de otras tipologías contractuales como son las becas, los contratos en prácticas y los contratos de formación, más alejados de la inserción laboral plena.

En lo que respecta al tipo de trabajo que han desarrollado, son relevantes las proporciones de universitarios que ocupan empleos como técnicos (en torno a un $50 \%$ ). Los comerciales y vendedores han disminuido en proporciones considerables, repercutiendo en un incremento significativo de los trabajadores del sector servicios y de la industria. No obstante, la pauta más destacada es la elevada proporción de universitarios que ocupan puestos de técnicos, acordes a su nivel de formación.

La principales vías de acceso al empleo han sido a través de amigos, familiares o conocidos y presentándose directamente. El sector informal adquiere relevancia a la hora de acceder al mercado laboral, siendo ésta una característica generalizada en el mercado de trabajo.

La situación laboral actual (julio de 2000) de estos universitarios se puede calificar de aceptable y similar a la de los que han desarrollado sus trayectorias profesionales en la empresa donde realizaron las prácticas. En concreto, la mayoría $(62,8 \%)$ desarrolla una actividad laboral; el tipo de contratación predominante es el contrato indefinido $(20,9 \%)$ y el temporal (36\%), frente a otras formas de contratación más precarias con menor representación, como becas y similares; y la categoría profesional que ostenta la mayoría $(62,1 \%)$ es la de técnico.

La proporción de universitarios que afirman no trabajar es minoritaria (37,2\%). Dentro de este grupo, algo más de la mitad $(56,7 \%)$ no busca trabajo, al preferir continuar su formación académica. De los que sí lo hacen, la mayoría (71\%) busca cualquier tipo de trabajo. Es relevante destacar que el $29 \%$ sólo busca un trabajo relacionado con las prácticas en empresas que realizó y, por tanto, relacionado con su formación académica (figura 5). 
FIGURA 5

Situación laboral actual (julio 2000)

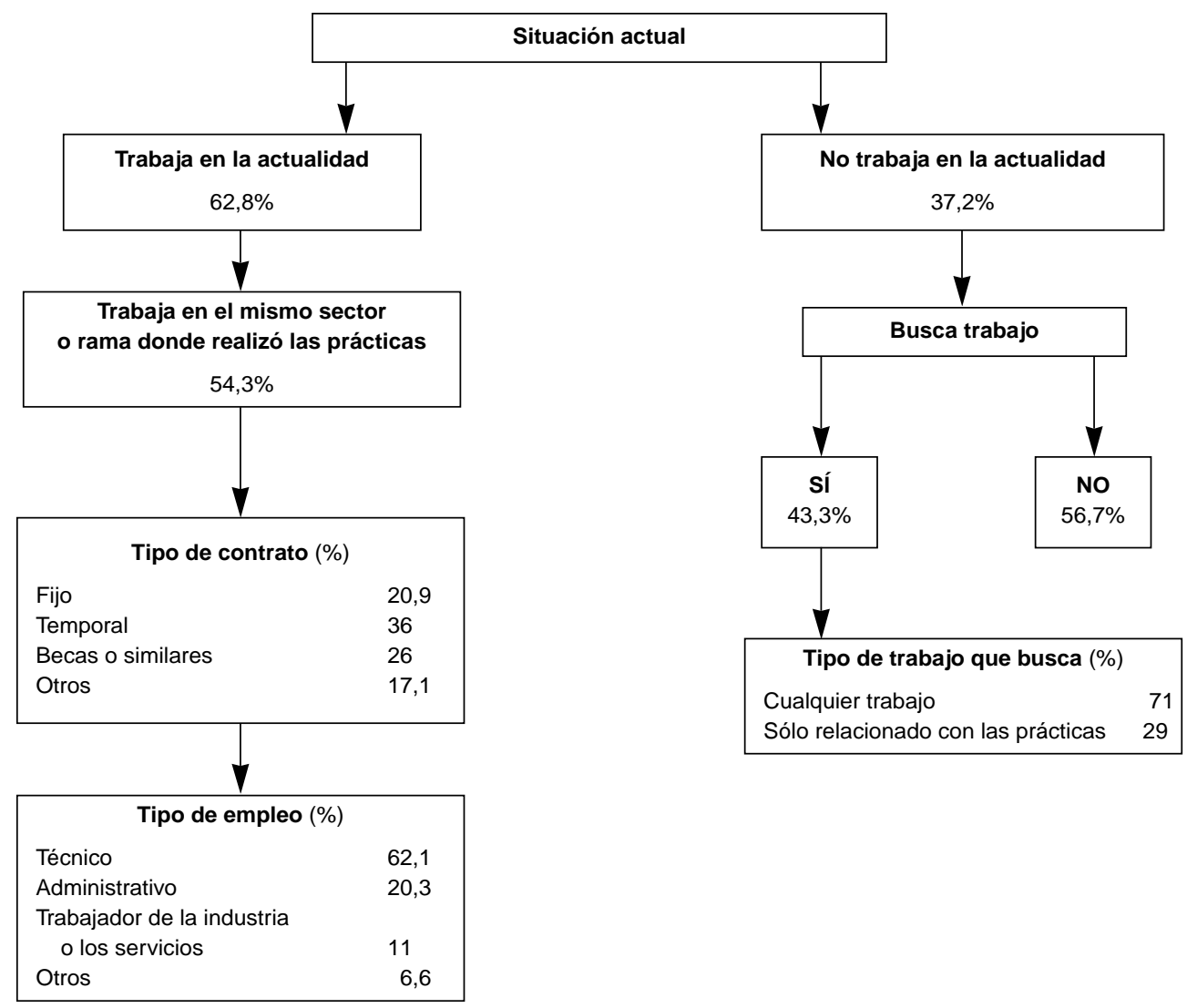

FUENTE:

Elaboración propia.

Los universitarios que emprendieron nuevas trayectorias laborales al abandonar la empresa donde realizaron las prácticas, en su mayoría consideran relevantes las cualificaciones que adquirieron durante el periodo de prácticas y afirman que han sido factores determinantes en sus procesos de inserción profesional. Por el contrario, otros aspectos, como las amistades, las posibilidades de promoción y la información que les ha proporcionado la experiencia práctica en empresas, creen que han tenido poca o ninguna relevancia en la con- 
figuración de su situación actual. No obstante, la mayoría (54,3\%) trabaja en la actualidad en el mismo sector o rama donde realizó las prácticas.

\section{PRINCIPALES VALORACIONES DE LOS UNIVERSITARIOS}

Los universitarios que han contado con la experiencia de realizar prácticas en empresas muestran un elevado grado de acuerdo (mucho o bastante) en que la experiencia laboral $(57,6 \%)$, la formación práctica $(57,9 \%)$ y la ampliación del currículum $(65,7 \%)$ que las prácticas les han proporcionado son factores relevantes para la inserción profesional. De hecho, la mayoría $(63,8 \%)$ las considera como una buena oportunidad de empleo.

Cuando se les pregunta por los seis factores más importantes para acceder al mercado laboral, en primer lugar señalan su iniciativa individual (19\%); le siguen, por orden de preferencia, la titulación cursada (17,5\%), las prácticas en empresas $(17 \%)$, la situación estructural del mercado de trabajo (16,5\%), el origen de clase social $(16 \%)$, y por último sitúan a la suerte ${ }^{13}(14 \%)$.

Las prácticas en empresas aparecen en tercer lugar, después de su propia iniciativa individual y de la titulación cursada.

Las valoraciones que realizan los universitarios acreditan la escasa importancia de factores como las amistades y la procedencia de clase en sus trayectorias laborales. De forma paradójica, cuando se les pregunta por las formas de acceso al trabajo, el sector informal (amigos, conocidos o familiares) adquiere relevancia por ser la principal vía de acceso al empleo.

Los diferentes estudios sobre inserción profesional (Iglesias y otros, 1997: 80; SOPP, 1996: 65; Navarro, 1989: 111; Fernández-Abascal, 1998: 182; González, 1998: 310; COIE, 1988: 75) coinciden a la hora de señalar como principal medio de acceso al empleo al sector informal, frente a las instituciones mediadoras entre desempleados y empleadores, que tienen escasa relevancia como vía de acceso al empleo; sin embargo, su protagonismo es incuestionable como instrumento de búsqueda.

\footnotetext{
13 Los jóvenes de Palencia consideran el factor «suerte» como determinante para encontrar un trabajo o mantenerlo. En concreto, el $45,5 \%$ está muy de acuerdo o de acuerdo con esta afirmación, frente a un $24,8 \%$ que no lo está y un $22,2 \%$ que se muestra indiferente (Fernández-Abascal, 1998: 182). Jenks et al. (1973; cit. por García, 1998: 15) se cuestionan la importancia de la educación en los logros laborales. En su trabajo concluían que la «suerte», como factor diferente de las características familiares, la educación y la herencia genética, explica en buena medida el status socioeconómico logrado y los ingresos.
} 


\section{CONCLUSIONES}

Las prácticas en empresas surgen con un objetivo claro de cubrir los déficit formativos de los universitarios. El ideal de buscar una formación práctica, in situ y adecuada a las demandas de los empresarios motiva que los poderes públicos las hayan impulsado en toda la Unión Europea.

La creciente implantación que han tenido en los años 1980 y su consolidación desde la década de 1990 han llevado a las universidades, conscientes de la necesidad de mejorarlas de forma continua, a evaluarlas en base a las cualificaciones que proporcionan a los universitarios y a la influencia que han tenido en sus procesos de transición a la vida activa.

Como se ha podido apreciar en las trayectorias profesionales descritas por los universitarios que las han realizado y en sus propias valoraciones, las prácticas en empresas han contribuido de forma significativa a la mejora de las cualificaciones y, en menor medida, a la consecución de una inserción profesional de calidad.

En la inmensa mayoría de los casos, no se cuestiona su eficacia como experiencia profesional ni como instrumento adecuado para la formación práctica; sin embargo, son reiteradas las dudas y las preguntas sobre las consecuencias que han podido tener en los procesos de inserción profesional.

El análisis de los resultados de distintas investigaciones (Cruz y otros, 1999; COIE, 1988; SOPP, 1996; Latiesa y otros, 1996) muestra que los universitarios que han realizado prácticas en empresas encuentran trabajo en mayor medida, tardan menos tiempo en encontrarlo y acceden a trabajos de mayor categoría, aunque con menor estabilidad.

Las prácticas son un buen medio de mejorar la formación de los universitarios al adquirir cualificaciones adecuadas a las demandas del mercado laboral. Ante el reto de la inserción laboral, estas cualificaciones los sitúan en una situación ventajosa, frente al resto de los universitarios que no las han realizado.

Las críticas y deficiencias también se han puesto de manifiesto ${ }^{14}$. En concreto, los universitarios las han calificado como «mano de obra barata», al proporcionar a los empresarios personal cualificado para realizar proyectos y cubrir necesidades bajo costes mínimos. Asi-

14 Según Trinidad y Pérez (1998: 5), los universitarios tienen una opinión negativa de las prácticas en empresas: «personas cualificadas a bajo precio». La concepción de que son mano de obra cualificada y barata es una de las opiniones más compartidas, aunque también destacan otros aspectos positivos de las prácticas, entre ellos la importancia de darse a conocer en las empresas como profesionales. 
mismo, consideran que los convenios firmados entre las universidades y las empresas suelen ser desconocidos para ellos, sin que puedan hacer valer sus derechos y obligaciones, quedando la situación controlada por aspectos informales.

Estos problemas son muy usuales y están motivados, en buena medida, por la falta de un tutor en la universidad, al ser una figura recomendada pero no obligatoria. Por el contrario, el tutor en la empresa es una figura obligatoria que existe en la mayoría de los casos, pero no siempre les proporciona toda la ayuda necesaria. Sobre todo en los conflictos que han surgido en las empresas, ya sea por la necesidad de acceder a información y material específico o por la propia relación con los compañeros de trabajo. En algunas ocasiones se han producido rechazos a los alumnos en prácticas, motivados por la competencia por los puestos de trabajo. El miedo de algunos empleados a perder el puesto de trabajo los ha provocado.

Los problemas que no responden a los fallos de la organización de las prácticas son difíciles de solventar. Sin embargo, la mejora de aspectos organizativos puede acabar con escollos que las mejorarían sustancialmente. En concreto, es fundamental que todos los convenios cuenten con un plan de trabajo adecuado a la actividad de la empresa y a la formación de los alumnos. En la mayoría de los casos $(55,2 \%)$, las empresas no contaban con un plan de trabajo, lo cual ha traído consigo algunas trabas que afectan de forma directa a la formación e inserción profesional de los alumnos.

La inadecuación entre la formación del universitario y las tareas que desempeñan durante las prácticas ha ocurrido en varias ocasiones. El desconocimiento de algunos empresarios sobre las competencias profesionales para las que preparan diferentes titulaciones ha hecho que muchos universitarios desempeñen cometidos inadecuados para su formación y, en otras ocasiones, alejados de sus posibilidades y de su responsabilidad.

La elaboración de un plan de trabajo previo, donde el tutor universitario participe activamente junto con el tutor en la empresa y el alumno, solventaría la práctica totalidad de estos problemas, que tienen solución e influyen directamente en la calidad de las prácticas.

Algunas titulaciones, entre ellas Sociología y Ciencias Políticas, tienen una notable utilidad para diferentes sectores empresariales, pero son totalmente desconocidas. El plan de trabajo ayudaría a dar a conocer a los empleadores las posibilidades de titulaciones con escasa implantación en la empresa privada.

Es necesaria una reforma de su regulación jurídica, dontándolas de mayor flexibilidad, donde los tutores en la empresa y en la universidad sean los verdaderos organizadores. 
La totalidad de las prácticas deben contar con financiación, con el objeto de cubrir los gastos que su realización suponen a los alumnos. Desde la Administración se han de articular medidas que favorezcan la continuidad laboral en la empresa, a través de exenciones fiscales para los empresarios, entre otros.

Por último, destacar la importancia de implicar cada vez más a los empresarios, a las universidades y a las administraciones, al ser una medida válida para la mejora de la formación práctica y un instrumento adecuado para facilitar la transición a la vida activa de los universitarios.

\section{BIBLIOGRAFÍA}

ASHFORTH, B., y SAKS, A. (1995): «Work-role transitions: A longitudinal examination of the Nicholson model», Journal of Occupational and Organizational Psychology, núm. 68, pp. 157-175.

AUBERNI, S. (comp) (1995): La orientación profesional, Institut Municipal d'educaciò, Barcelona.

BECKER, G. S. (1983): «Inversión en capital humano e ingresos», en L. Toharia, El mercado de trabajo: teorías explicativas, Alianza, Madrid, pp. 39-64.

BLANCH, J. M. (1990): Del viejo al nuevo paro, PPU, Barcelona.

CASAL, J. (1997): Modalidades de transición profesional, mercado de trabajo y condiciones de empleo, Jornadas sobre Inserción Laboral, GRET, ICE-UAB, 27 y 28 de noviembre.

CARABAÑA, J. (1999): Dos estudios sobre movilidad intergeneracional, Fundación Argentaria-Visor, Madrid.

COIE (Centro de Orientación e Información al Empleo) (1988): Estudio sobre la inserción laboral de los titulados por la Universidad de Granada, Vicerrectorado de Estudiantes, Universidad de Granada, Granada. Inédito.

- (1995): El desempleo de larga duración entre los titulados universitarios, Vicerrectorado de Estudiantes, Universidad de Granada, Granada.

- (1997): La tendencia de la oferta de empleo en las empresas de Granada. Una aproximación a las carencias formativas de la población activa universitaria y a las posibilidades latentes de creación de empleo en la provincia, Vicerrectorado de Estudiantes, Universidad de Granada, Granada.

CRUZ, F., y otros (1999): Universidad y Sociedad, Universidad de Huelva, Consejo Social, Huelva.

DOERINGER, P. B., y PIORE, M. J. (1983a): «El paro y el mercado de trabajo», en L. Toharia, El mercado de trabajo: teorías explicativas, Alianza, Madrid, pp. 307-339.

- (1983b): «Los mercados internos de trabajo», en L. Toharia, El mercado de trabajo: teorías explicativas, Alianza, Madrid, pp. 341-388.

FERNÁNDEZ-ABASCAL, H. (1998) (coord.): Condiciones y oportunidades de empleo de los jóvenes de Palencia, Escuela Universitaria de Relaciones Laborales, Palencia.

FIGUERA, P. (1996): La inserción del universitario en el mercado de trabajo, PPU, EUB, Barcelona. 
GARCíA, J. L. (2000) (dir.): El libro blanco de la formación, Cívitas, Madrid.

GARCÍA, J., y PASTOR, M. S. (1999): «Guía para una buena práctica de las prácticas en empresas», en Guía de las empresas que ofrecen empleo, Fundación Universidad-Empresa, Madrid.

GARCÍA, M. a I. (1998): Recursos formativos e inserción laboral de los jóvenes, CIS, Madrid.

GARCÍA, J. (1998a): The dualisation of unemployment risks class and insider/outsider patterns in the spanish labour market, Instituto Juan March de Estudios e Investigaciones, Madrid.

GONZÁLEZ, J. M. (1998): Las actitudes de los jóvenes universitarios hacia las nuevas demandas del mercado laboral, Tesis doctoral inédita, Universidad de Granada.

INCYDE (2001): La creación de empresas en España, Cámaras de Comercio e Industria de España, Madrid.

IGLESIAS, J., y otros. (1997): Sociología de una profesión: los astrónomos en España, Working Paper, Facultad de Ciencias Políticas y Sociología, Universidad de Granada, Granada.

LATIESA, M.; NÚÑEZ, J., y MARTíNEZ, R. (2001): Políticas y Sociología: ámbitos académico y laboral, Universidad de Granada, Granada.

LATIESA, M., y otros (1996): Trayectorias académicas y profesionales de los licenciados en Ciencias Políticas y Sociología, Working Paper, Facultad de Ciencias Políticas y Sociología, Universidad de Granada, Granada.

LÓPEZ, A., y HERNÁNDEZ, J. (2001) (comps.): Jóvenes más allá del empleo, Nau Libres, Valencia.

MARTÍN, M., y VELARDE, O. (2001): Informe Juventud en España 2000, Ministerio de Trabajo y Asuntos Sociales, Instituto de la Juventud, Madrid.

MARTÍNEZ, R. (2002): La inserción laboral de los universitarios, Universidad de Granada, Granada.

MARTÍNEZ, A. M. a , y VALERO, F. J. (1999): Evaluación del impacto de las prácticas en empresas, Vicerrectorado de Fomento y Relaciones Universidad-Empresa, Universidad de Granada.

MASJUAN, J. M., y otros. (1996): La inserción professional dels nous titulats universitaris, ICE, UAB, Barcelona.

MTAS (Ministerio de Trabajo y Asuntos Sociales) (1998): El mercado laboral de los titulados universitarios, INEM, Madrid.

NAVARRO, J. (dir. ) (1989): Las situaciones y perfil del desempleo y subempleo de los titulados universitarios, Consejo de Universidades, Madrid.

OCDE (Organización para la Cooperación y el Desarrollo Económico) (1998): Perspectivas de empleo 1998, Ministerio de Trabajo y Asuntos Sociales, Madrid.

OIT (Oficina Internacional del Trabajo) (1998): Informe sobre el empleo en el mundo 1998-1999, Ginebra.

POOLE, M. E.; LANGAN-FOX, J., y OMODEI, M. (1993): «Contrasting subjetive and objetive criteria as determinants of perceived career success: A longitudinal study», Journal of Occupational and Organizational Psychology, núm. 66, pp. 39-54.

RANDOM (2001): Trayectorias laborales de los diplomados en Fisioterapia, Consejo Social, Universidad Complutense, Madrid.

- (2001a): Trayectorias laborales de los diplomados en Enfermería, Consejo Social, Universidad Complutense, Madrid. 
RANDOM (2001b): Trayectorias laborales de los diplomados en Óptica, Consejo Social, Universidad Complutense, Madrid.

- (2001c): Trayectorias laborales de los diplomados en Biblioteconomía y Documentación, Consejo Social, Universidad Complutense, Madrid.

- (2001d): Trayectorias laborales de los diplomados en Podología, Consejo Social, Universidad Complutense, Madrid.

- (2001e): Trayectorias laborales de los diplomados en Empresariales, Consejo Social, Universidad Complutense, Madrid.

REQUENA, F. (1991): Redes sociales y mercado de trabajo, CIS, Madrid.

RETUERTO, E. (1997): «El nuevo enfoque de las competencias profesionales y el aprendizaje a lo largo de toda la vida activa», Economía y Sociología del Trabajo, Ministerio de Trabajo y Asuntos Sociales, Madrid, núm. 1, pp. 103-115.

RIVIÉRE, J. (1997): «La formación como ocupación y como adquisición de capital humano», en L. Garrido y M. Requena, La emancipación de los jóvenes en España, Ministerio de Trabajo y Asuntos Sociales, Instituto de la Juventud, pp. 85-114.

SALVÁ, F. (2000) (dir.): Formación e Inserción laboral, Pirámide, Madrid.

SOPP (Servicio de Orientación y Planificación Profesional) (1996): Estudio de inserción laboral de los titulados de la Universidad Carlos III de Madrid, Fundación Universidad Carlos III, Madrid.

TRINIDAD, A., y PÉREZ, M. (1998): Evaluación de la Facultad de Ciencias Políticas y Sociología de Granada, Universidad de Granada, Facultad de Políticas y Sociología, Granada.

\section{ABSTRACT}

The practices in companies for college students are regulated in 1981(RD 1491/1981) with the objective to improve the practical formation of the students of last courses of race. Its implantation is a reality in the Nineties with the implication every greater time of institutions, industralists and students, who respond to the necessity to approach it university the enterprise world and to facilitate the labor insertion of the college students. In the present analyse article the practical experience in the labor company, university opinions and of the valuations on the practices in companies in the University of Granada (Spain) and its trajectories. 